\title{
Weight management of a patient with fourth cervical and fifth cervical vertebrae spinal cord injury: a case study
}

\author{
S. S. Wong ${ }^{1,2}$, A. Graham ${ }^{3}$, A. Forbes ${ }^{2} \&$ G. Grimble ${ }^{2}$ \\ ${ }^{1}$ Department of Nutrition and Dietetics, Stoke Mandeville Hospital, Aylesbury, UK, ${ }^{2}$ Centre for Gastroenterology and \\ Clinical Nutrition, University College London, London, UK, ${ }^{3}$ National Spinal Injuries Centre, Stoke Mandeville Hospital, \\ Aylesbury, UK
}

Obesity in the patient with spinal cord injuries (SCI) is an ongoing clinical and public health problem that often goes undetected and unmanaged $^{(1)}$. It is associated with significant adverse health outcomes and healthcare expenditure ${ }^{(2)}$. In 2006 the National Institute for Health and Clinical Excellence published guidance in managing overweight and obesity. It is known that the inherent immobility and muscle atrophy associated with SCI emphasise the risk of overnutrition. However, to date there is no weight management clinic operating in the UK spinal injury centres. This report presents a case study of a 43-year-old man with fourth cervical and fifth cervical vertebrae incomplete spinal cord injury (grade C of the American Spinal Injury Association Impairment Scale ${ }^{(3)}$ ) after a road traffic accident 25 years ago and a known family history of metabolic syndrome and dyslipidaemia, who was referred to the National Spinal Injury Centre specialist weight management outpatient clinic for dietetic advice covering nutrition, exercise and cognitive behaviour therapy. A general healthy dietary advice based on the eatwell plate ${ }^{(4)}$ is discussed in the initial appointment. If weight loss plateaus in the subsequent follow up, an $2.5 \mathrm{MJ}(600 \mathrm{kcal})$ deficit diet is given to patients. Various outcomes are measured: anthropometric measurements including BMI, mid upper arm circumference (MUAC), triceps skinfold thickness (TSF), mid-arm muscle circumference (MAMC); lipid profile including total cholesterol, LDL, HDL and TAG; dietary assessment by $7 \mathrm{~d}$ food diary.

The subject was classified as grade I obese ${ }^{(2,5)}$ at the initial visit to the clinic: weight $111.1 \mathrm{~kg}$; BMI $34.2 \mathrm{~kg} / \mathrm{m}^{2}$; MUAC $35.1 \mathrm{~cm}$; TSF $37.2 \mathrm{~mm}$; MAMC $23.4 \mathrm{~cm}$. In the subsequent follow up there were significant reductions (at weeks 4 and 12 respectively) in weight (4.1 kg ( $3.7 \%$ weight loss) and $13.4 \mathrm{~kg}(12.1 \%$ weight loss) $)$ BMI $\left(33 \mathrm{~kg} / \mathrm{m}^{2}\right.$ and $\left.30.1 \mathrm{~kg} / \mathrm{m}^{2}\right)$, MUAC $(35 \mathrm{~cm}$ and $34.3 \mathrm{~cm})$ and TSF $(36.8 \mathrm{~mm}$ and $34.2 \mathrm{~mm}$ ). It was noted that lean body mass was maintained throughout. MAMC was $23.4 \mathrm{~cm}$ at 4 weeks, increasing to $23.5 \mathrm{~cm}$ at 8 weeks. Blood biochemistry was measured at week 4 and week 12 after initial consultation as per local weight management protocol. The subject showed an improvement in lipid profile, with reductions (at weeks 4 and 12 respectively) in total cholesterol $(5.5 \mathrm{mmol} / \mathrm{l}$ and $4.5 \mathrm{mmol} / \mathrm{l}$; $18 \%)$, LDL $(2.5 \mathrm{mmol} / \mathrm{l}$ and $2.2 \mathrm{mmol} / \mathrm{l} ; 12 \%)$ and TAG $(4.3 \mathrm{mmol} / \mathrm{l}$ and $3.4 \mathrm{mmol} / \mathrm{l} ; 20 \%)$. HDL-cholesterol was maintained at $0.7 \mathrm{mmol} / \mathrm{l}$. A $7 \mathrm{~d}$ food diary analysis showed that average daily intake was reduced from $17.2 \mathrm{MJ}$ (4100 kcal) at 0 weeks (week prior to appointment) to $6.28 \mathrm{MJ}(1500 \mathrm{kcal})$ at 12 weeks (week prior to appointment). The subject's clinical condition was stable and the follow up will be continued by regular attendance at the weight management clinic.

The present report describes the course of successful weight management for a patient with SCI in relation to nutritional requirement, body composition and the consequence of overnutrition. Presentation of this case could be useful to healthcare professionals in dealing with similar cases.

The project was support by an unrestricted grant from Sanofi-Aventis.

1. Gupta N, White K \& Sandford P (2006) Spinal Cord 44, 92-94.

2. National Institute for Health and Clinical Excellence (2006) Guidance on the Prevention, Identification, Assessment and Management of Overweight and Obesity in Adults and Children. London: NICE.

3. Marino RJ, Barros T, Biering-Sorensen F et al. (2003) J Spinal Cord Med 26, S50-S56.

4. Food Standard Agency (2009) The eatwell plate. www.food.gov.uk (assessed April 2009)

5. World Health Organization (2000) Obesity: Preventing and Managing the Global Epidemic. Report of a WHO Consultation. WHO Technical Report Series 894. Geneva: WHO. 